PROCEEDINGS OF THE

AMERICAN MATHEMATICAL SOCIETY

Volume 128, Number 8 , Pages $2357-2363$

S 0002-9939(99)05335-6

Article electronically published on December 7, 1999

\title{
RELATIONS BETWEEN THE TAYLOR SPECTRUM AND THE XIA SPECTRUM
}

\author{
MUNEO CHŌ \\ (Communicated by David R. Larson) \\ Dedicated to Professor Jyunji Inoue on his sixtieth birthday
}

\begin{abstract}
Let $\mathbf{T}=\left(T_{1}, T_{2}, \ldots, T_{n}\right)$ be a doubly commuting $n$-tuple of $p$ hyponormal operators $T_{j}$ with unitary operators $U_{j}$ from the polar decompositions $T_{j}=U_{j}\left|T_{j}\right|(j=1, \ldots, n)$. Let $\mathbf{U}=\left(U_{1}, \ldots, U_{n}\right)$ and $A=\left|T_{1}\right| \cdots\left|T_{n}\right|$. In this paper, we will show relations between the Taylor spectrum $\sigma_{T}(\mathbf{T})$ and the Xia spectrum $\sigma_{X}(\mathbf{U}, A)$.
\end{abstract}

\section{INTRODUCTION}

In [12, D. Xia introduced a class of semi-hyponormal tuples and a notion of spectrum for such tuples. We call this spectrum the Xia spectrum. Xia proved Putnam's inequality for semi-hyponormal tuples. In [6], M. Chō and T. Huruya generalized Putnam's inequality to $p$-hyponormal tuples. Also, in 9], B. P. Duggal showed a very interesting inequality of doubly commuting $n$-tuples of $p$-hyponormal operators. In this paper, we show that the Xia spectrum of a doubly commuting $n$-tuple $\mathbf{T}=\left(T_{1}, \ldots, T_{n}\right)$ of $p$-hyponormal operators $T_{j}$ with unitary operators $U_{j}$ from the polar decompositions $T_{j}=U_{j}\left|T_{j}\right|(j=1, \ldots, n)$ essentially coincides with its Taylor spectrum.

Let $\mathcal{H}$ be a complex separable Hilbert space and $B(\mathcal{H})$ the set of all bounded linear operators on $\mathcal{H}$. For $T \in B(\mathcal{H})$, let $\sigma(T)$ be the spectrum of $T$. An operator $T \in B(\mathcal{H})$ is called $p$-hyponormal if $\left(T^{*} T\right)^{p} \geq\left(T T^{*}\right)^{p}$. If $p=\frac{1}{2}$, then $T$ is called semi-hyponormal. Let $W$ be a unitary operator and $A \in B(\mathcal{H})$. If

$$
\mathcal{S}_{W}^{ \pm}(A)=\mathrm{s}-\lim _{n \rightarrow \pm \infty}\left(W^{-n} A W^{n}\right)
$$

exist, then the operators $\mathcal{S}_{W}^{ \pm}(A)$ are called the polar symbols of $A$ (with respect to $W$ ). Let $T=U|T|$ be the polar decomposition of $T$. If $T$ is semi-hyponormal and $U$ is unitary, then $\mathcal{S}_{U}^{ \pm}(|T|)$ exist (cf. [13]). In [13, D. Xia proved the following theorem:

Received by the editors March 12, 1998 and, in revised form, September 18, 1998.

1991 Mathematics Subject Classification. Primary 47B20.

Key words and phrases. Putnam's inequality, Taylor spectrum, Xia spectrum, generalized polar symbols.

This research was partially supported by Grant-in-Aid Scientific Research No.09640229. 
Theorem A (Theorem IV.4.1 of [13]). Let $T=U|T|$ be a semi-hyponormal operator with $U$ unitary. Then

$$
\sigma(T)=\bigcup_{0 \leq k \leq 1} \sigma\left(T_{(k)}\right),
$$

where $T_{(k)}=k \mathcal{S}_{U}^{+}(T)+(1-k) \mathcal{S}_{U}^{-}(T)$.

\section{Generalized polar symbols}

Throughout this paper let $p$ be such that $0<p<\frac{1}{2}$. Let $T=U|T|$ be a $p$ hyponormal operator with $U$ unitary. Since $U|T|^{2 p}$ is semi-hyponormal, there exist $\mathcal{S}_{U}^{ \pm}\left(|T|^{2 p}\right)$. For $0 \leq k \leq 1$, we denote

$$
T_{k}=U\left\{k \mathcal{S}_{U}^{+}\left(|T|^{2 p}\right)+(1-k) \mathcal{S}_{U}^{-}\left(|T|^{2 p}\right)\right\}^{\frac{1}{2 p}} ;
$$

we call the operators $T_{k}$ the generalized polar symbols of $T$. Note that if an operator $T=U|T|$ is a semi-hyponormal operator with $U$ unitary, then $T_{(k)}=T_{k}$ for every $0 \leq k \leq 1$. It is easy to check that $T_{k}$ is a normal operator for every $0 \leq k \leq 1$. For $T \in B(\mathcal{H})$, let $\sigma_{n a}(T)$ denote the normal approximate point spectrum of $T$, i.e., the set of all complex numbers $z$ which satisfy the following condition: there exists a sequence $\left\{x_{n}\right\}$ of unit vectors in $\mathcal{H}$ such that

$$
\lim _{n \rightarrow \infty}\left\|(T-z) x_{n}\right\|=\lim _{n \rightarrow \infty}\left\|(T-z)^{*} x_{n}\right\|=0 .
$$

If $T$ is a normal operator, then $\sigma(T)=\sigma_{n a}(T)$. In 12 this spectrum is called the joint approximate point spectrum, but we use this term for $n$-tuple of operators. The following theorem holds.

Theorem B (Lemma I.2.4 of [13]). Let $T \in B(\mathcal{H})$ and let $T=U|T|$ be the polar decomposition of $T$. Let $r>0$. Then $r e^{i \theta} \in \sigma_{n a}(T)$ if and only if there exists a sequence $\left\{x_{n}\right\}$ of unit vectors in $\mathcal{H}$ such that

$$
\lim _{n \rightarrow \infty}\left\|(|T|-r) x_{n}\right\|=\lim _{n \rightarrow \infty}\left\|\left(U-e^{i \theta}\right) x_{n}\right\|=0 .
$$

Therefore, for a semi-hyponormal operator $T=U|T|$ with $U$ unitary and a nonzero $r e^{i \theta} \in \mathbf{C}$, it follows that $r e^{i \theta} \in \sigma_{n a}\left(T_{(k)}\right)$ if $r e^{i \theta} \in \sigma\left(T_{(k)}\right)$, because each $T_{(k)}$ is a normal operator $(0 \leq k \leq 1)$.

The following result was proved in [5]. For the sake of completeness, we will give a simple proof.

Theorem 1 (Theorem of [5). Let $T=U|T|$ be a p-hyponormal operator with $U$ unitary. Then

$$
\sigma(T)=\bigcup_{0 \leq k \leq 1} \sigma\left(T_{k}\right) .
$$

For the proof of this theorem, we need the following result.

Theorem C (Theorem 3 of [4]). Let $T=U|T|$ be a p-hyponormal operator with $U$ unitary. Then

$$
\sigma\left(U|T|^{2 p}\right)=\left\{r^{2 p} e^{i \theta} \mid r e^{i \theta} \in \sigma(T)\right\} .
$$

Proof of Theorem 1. Note that $\mathcal{S}_{U}^{-}\left(|T|^{2 p}\right) \leq|T|^{2 p} \leq \mathcal{S}_{U}^{+}\left(|T|^{2 p}\right)$ (cf. Th.II.2.7 of [13]). If $0 \in \sigma(T)$, then $0 \in \sigma(|T|)$ and hence $0 \in \sigma\left(T_{0}\right)\left(T_{0}=U\left\{\mathcal{S}_{U}^{-}\left(|T|^{2 p}\right)\right\}^{\frac{1}{2 p}}\right)$.

Conversely, let $0 \in \bigcup_{0 \leq k \leq 1} \sigma\left(T_{k}\right)$. Since $T_{k}$ is normal, we have $0 \in \sigma\left(\mathcal{S}_{U}^{-}\left(|T|^{2 p}\right)\right)$ and 
hence $0 \in \sigma(|T|)$ (cf. Th.II.1.5 of [12]). Therefore, we have $0 \in \sigma(T)$. Next we prove that, for a non-zero $z=r e^{i \theta} \in \mathbf{C}, z \in \sigma(T)$ if and only if $z \in \bigcup_{0<k \leq 1} \sigma\left(T_{k}\right)$.

Let $S=U|T|^{2 p}$. Then $S$ is semi-hyponormal and from Theorem [C] we have

$$
\begin{gathered}
z \in \sigma(T) \Longleftrightarrow r^{2 p} e^{i \theta} \in \sigma(S) \\
\Longleftrightarrow \exists k(0 \leq k \leq 1) ; r^{2 p} e^{i \theta} \in \sigma\left(S_{(k)}\right) \quad \text { (from Theorem A) } \\
\Longleftrightarrow \exists k(0 \leq k \leq 1) ; r^{2 p} e^{i \theta} \in \sigma_{n a}\left(S_{(k)}\right) \quad \text { (from Theorem B) } \\
\Longleftrightarrow \exists k(0 \leq k \leq 1) ; r e^{i \theta} \in \sigma_{n a}\left(T_{k}\right) \\
\Longleftrightarrow z \in \bigcup_{0 \leq k \leq 1} \sigma\left(T_{k}\right) .
\end{gathered}
$$

The proof is now complete.

\section{The Taylor spectrum and the Xia spectrum}

For a commuting $n$-tuple $\mathbf{T}=\left(T_{1}, \ldots, T_{n}\right)$, the Taylor spectrum and the joint approximate point spectrum of $\mathbf{T}$ are denoted by $\sigma_{T}(\mathbf{T})$ and $\sigma_{j a}(\mathbf{T})$, respectively. It is well known that $\sigma_{T}(\mathbf{T})=\sigma_{j a}(\mathbf{T})$ if $\mathbf{T}$ is a commuting $n$-tuple of normal operators. If $\mathbf{T}=\left(T_{1}, \ldots, T_{n}\right)$ is a doubly commuting $n$-tuple of $p$-hyponormal operators, then, by Theorem 7 of [3], it follows that $\sigma_{T}(\mathbf{T})=\left\{\left(z_{1}, \ldots, z_{n}\right) \in \mathbf{C}^{n}:\left(\overline{z_{1}}, \ldots, \overline{z_{n}}\right) \in\right.$ $\left.\sigma_{j a}\left(\mathbf{T}^{*}\right)\right\}$, where $\mathbf{T}^{*}=\left(T_{1}^{*}, \ldots, T_{n}^{*}\right)$. Let $\mathbf{U}=\left(U_{1}, \ldots, U_{n}\right)$ be a commuting $n$-tuple of unitary operators. Let $\mathbf{Q}_{j}(j=1, \ldots, n)$ on $B(\mathcal{H})$ be defined by

$$
\mathbf{Q}_{j} A=A-U_{j} A U_{j}^{*} \quad(A \in B(\mathcal{H})) .
$$

Let $A \in B(\mathcal{H})$ and $A \geq 0$. An $(n+1)$-tuple $(\mathbf{U}, A)$ is called $p$-hyponormal if

$$
\mathbf{Q}_{j_{1}} \cdots \mathbf{Q}_{j_{m}} A^{2 p} \geq 0
$$

for all $1 \leq j_{1}<\cdots<j_{m} \leq n$. We simply denote $\mathcal{S}_{U_{j}}^{ \pm}(A)$ by $\mathcal{S}_{j}^{ \pm}(A)$ for every $j=1, \ldots, n$. Let $(\mathbf{U}, A)$ be a $p$-hyponormal tuple and $0 \leq k \leq 1$. We denote

$$
\left(k \mathcal{S}_{j}^{+}+(1-k) \mathcal{S}_{j}^{-}\right)_{p} A=\left\{k \mathcal{S}_{j}^{+}\left(A^{2 p}\right)+(1-k) \mathcal{S}_{j}^{-}\left(A^{2 p}\right)\right\}^{\frac{1}{2 p}} .
$$

For $\mathbf{k}=\left(k_{1}, \ldots, k_{n}\right) \in[0,1]^{n}$, the general polar symbols $A_{\mathbf{k}}$ of $A$ are defined by

$$
A_{\mathbf{k}}=\prod_{j=1}^{n}\left(k_{j} \mathcal{S}_{j}^{+}+\left(1-k_{j}\right) \mathcal{S}_{j}^{-}\right)_{p} A .
$$

Then, by 6$],\left(\mathbf{U}, A_{\mathbf{k}}\right)$ is a commuting $(n+1)$-tuple of normal operators for every $\mathbf{k} \in[0,1]^{n}$. We define the Xia spectrum $\sigma_{X}(\mathbf{U}, A)$ of $(\mathbf{U}, A)$ by

$$
\sigma_{X}(\mathbf{U}, A)=\bigcup_{\mathbf{k} \in[0,1]^{n}} \sigma_{j a}\left(\mathbf{U}, A_{\mathbf{k}}\right) .
$$

By Theorem 2 of [6] it follows that, for a $p$-hyponormal tuple $(\mathbf{U}, A)$,

$$
\left\|\mathbf{Q}_{1} \cdots \mathbf{Q}_{n} A^{2 p}\right\| \leq \frac{2 p}{(2 \pi)^{n}} \int \cdots \int_{\sigma_{X}(\mathbf{U}, A)} r^{2 p-1} d \theta_{1} \cdots d \theta_{n} d r .
$$

We now have the following

Lemma 2. Let $\mathbf{T}=\left(T_{1}, \ldots, T_{n}\right)$ be a doubly commuting $n$-tuple of -hyponormal operators $T_{j}=U_{j}\left|T_{j}\right|$ with $U_{j}$ unitary operators $(j=1, \ldots, n)$, and let $\mathbf{U}=\left(U_{1}, \ldots, U_{n}\right)$ and $A=\left|T_{1}\right| \cdots\left|T_{n}\right|$. Then $(\mathbf{U}, A)$ is p-hyponormal. 
Proof. Since $A^{2 p}=\left|T_{1}\right|^{2 p} \cdots\left|T_{n}\right|^{2 p}$, we have

$$
\mathbf{Q}_{j} A^{2 p}=\left(\prod_{i \neq j}\left|T_{i}\right|^{2 p}\right)\left(\left|T_{j}\right|^{2 p}-U_{j}\left|T_{j}\right|^{2 p} U_{j}^{*}\right)
$$

for every $j(j=1, \ldots, n)$. Hence $(\mathbf{U}, A)$ is $p$-hyponormal.

With the above notations (Lemma 21), we also have, using the above,

$$
\left\|\prod_{j=1}^{n}\left(\left|T_{j}\right|^{2 p}-\left|T_{j}^{*}\right|^{2 p}\right)\right\| \leq \frac{2 p}{(2 \pi)^{2 n}} \int \cdots \int_{\sigma_{X}(\mathbf{U}, A)} r^{2 p-1} d \theta_{1} \cdots d \theta_{n} d r
$$

(this inequality is due to Duggal [9]), and

$$
\left(k_{j} \mathcal{S}_{j}^{+}+\left(1-k_{j}\right) \mathcal{S}_{j}^{-}\right)_{p} A=\left(\prod_{i \neq j}\left|T_{i}\right|\right)\left\{k_{j} \mathcal{S}_{j}^{+}\left(\left|T_{j}\right|^{2 p}\right)+\left(1-k_{j}\right) \mathcal{S}_{j}^{-}\left(\left|T_{j}\right|^{2 p}\right)\right\}^{\frac{1}{2 p}} .
$$

Hence, for every $\mathbf{k}=\left(k_{1}, \ldots, k_{n}\right) \in[0,1]^{n}$, it follows that

$$
A_{\mathbf{k}}=\prod_{j=1}^{n} A_{j},
$$

where $A_{j}=\left\{k_{j} \mathcal{S}_{j}^{+}\left(\left|T_{j}\right|^{2 p}\right)+\left(1-k_{j}\right) \mathcal{S}_{j}^{-}\left(\left|T_{j}\right|^{2 p}\right)\right\}^{\frac{1}{2 p}} \quad(j=1, \ldots, n)$. We prove the following

Theorem 3. Let $\mathbf{T}=\left(T_{1}, \ldots, T_{n}\right)$ be a doubly commuting n-tuple of p-hyponormal operators with unitary operators $U_{j}$ from the polar decompositions $T_{j}=U_{j}\left|T_{j}\right| \quad(j=$ $1, \ldots, n)$. Let $\mathbf{U}=\left(U_{1}, \ldots, U_{n}\right)$ and $A=\left|T_{1}\right| \cdots\left|T_{n}\right|$. If $\left(z_{1}, \ldots, z_{n}, a\right) \in \sigma_{X}(\mathbf{U}, A)$, then there exist non-negative numbers $a_{1}, \ldots, a_{n}$ such that $\left(z_{1} a_{1}, \ldots, z_{n} a_{n}\right) \in \sigma_{T}(\mathbf{T})$ and $a=a_{1} \cdots a_{n}$.

Conversely, if $\left(z_{1} a_{1}, \ldots, z_{n} a_{n}\right) \in \sigma_{T}(\mathbf{T})$, then $\left(z_{1}, \ldots, z_{n}, a_{1} \cdots a_{n}\right) \in \sigma_{X}(\mathbf{U}, A)$, where $\left|z_{j}\right|=1$ and $a_{j} \geq 0$ for every $j(j=1, \ldots, n)$.

For the proof of this theorem, we need the following Berberian extension theorem.

Theorem $\mathbf{D}$ (Theorem 1 of 1$]$ ). Let $B(\mathcal{H})$ be the algebra of all bounded operators on $\mathcal{H}$. Then there exist an extension space $\mathcal{K}$ of $\mathcal{H}$ and a faithful ${ }^{*}$-representation of $B(\mathcal{H})$ into $B(\mathcal{K}): T \rightarrow T^{\circ}$ such that

$$
\sigma_{j a}\left(T_{1}, \ldots, T_{n}\right)=\sigma_{j a}\left(T_{1}^{\circ}, \ldots, T_{n}^{\circ}\right)=\sigma_{p}\left(T_{1}^{\circ}, \ldots, T_{n}^{\circ}\right),
$$

where $\sigma_{p}\left(T_{1}, \ldots, T_{n}\right)$ is the joint point spectrum of $\left(T_{1}, \ldots, T_{n}\right)$. Moreover, if $T$ is p-hyponormal, then $T^{\circ}$ is also p-hyponormal.

Proof of Theorem 3 . First we assume that $\left(z_{1}, \ldots, z_{n}, a\right) \in \sigma_{X}(\mathbf{U}, A)$. We show by induction that there exist $a_{1}, \ldots, a_{n}\left(\forall a_{j} \geq 0\right)$ such that

$$
\left(z_{1} a_{1}, \ldots, z_{n} a_{n}\right) \in \sigma_{T}(\mathbf{T}) \text { and } a=a_{1} \cdots a_{n} .
$$

If $n=1$, Theorem 3 holds by Theorem 3 of [6]. By inductive hypothesis, there exist $\mathbf{k}=\left(k_{1}, \ldots, k_{n}\right) \in[0,1]^{n}$ and a sequence $\left\{x_{m}\right\}$ of unit vectors such that

$$
\left(U_{j}-z_{j}\right) x_{m} \rightarrow 0(j=1, \ldots, n) \text { and }\left(A_{\mathbf{k}}-a\right) x_{m} \rightarrow 0,
$$

where $A_{\mathbf{k}}=\prod_{j=1}^{n}\left(k_{j} \mathcal{S}_{j}^{+}+\left(1-k_{j}\right) \mathcal{S}_{j}^{-}\right)_{p} A$. By Lemma 2 we have

$$
A_{\mathbf{k}}=\prod_{j=1}^{n} A_{j},
$$


where $A_{j}=\left\{k_{j} \mathcal{S}_{j}^{+}\left(\left|T_{j}\right|^{2 p}\right)+\left(1-k_{j}\right) \mathcal{S}_{j}^{-}\left(\left|T_{j}\right|^{2 p}\right)\right\}^{\frac{1}{2 p}}$. By Theorem $\mathrm{D}$ let $\mathcal{K}$ be the extension space of $\mathcal{H}$. Then

$$
\mathcal{M}=\operatorname{Ker}\left(U_{1}^{\circ}-z_{1}\right) \cap \cdots \cap \operatorname{Ker}\left(U_{n}^{\circ}-z_{n}\right) \cap \operatorname{Ker}\left(A_{\mathbf{k}}^{\circ}-a\right)
$$

is a non-zero subspace of $\mathcal{K}$. Since $\left(U_{1}^{\circ}, \ldots, U_{n}^{\circ}, A_{1}^{\circ}, \ldots, A_{n}^{\circ}\right)$ is a commuting $2 n$-tuple, $\mathcal{M}$ is an invariant subspace for $A_{1}^{\circ}, \ldots, A_{n}^{\circ}$. Also since $a \in \sigma\left(A_{\mathbf{k} \mid \mathcal{M}}^{\circ}\right)$, there exist $a_{1}, \ldots, a_{n}$ and a non-zero vector $x^{\circ} \in \mathcal{M}$ such that

$$
\left(A_{j}^{\circ}-a_{j}\right) x^{\circ}=0 \text { for every } j(j=1, \ldots, n) \text { and } a=a_{1} \cdots a_{n},
$$

by Theorem $\mathrm{D}$ and the spectral mapping theorem for the joint spectrum. Let

$$
\mathcal{N}=\operatorname{Ker}\left(U_{n}^{\circ}-z_{n}\right) \cap \operatorname{Ker}\left(A_{n}^{\circ}-a_{n}\right) .
$$

Then

$$
\left(z_{1}, \ldots, z_{n-1}, a_{1} \cdots a_{n-1}\right) \in \sigma_{X}\left(\mathbf{U}^{\prime}, \mathbf{A}^{\prime}\right)
$$

where $\mathbf{U}^{\prime}=\left(U_{1}, \ldots, U_{n-1}\right)$ and $\mathbf{A}^{\prime}=\prod_{j=1}^{n-1} A_{j}$. By Theorem $\mathbb{D}$ and the inductive hypothesis, we have

$$
\left(z_{1} a_{1}, \ldots, z_{n-1} a_{n-1}\right) \in \sigma_{T}\left(T_{1}, \ldots, T_{n-1}\right) .
$$

Since $\mathbf{S}=\left(T_{1 \mid \mathcal{N}}^{\circ}, \ldots, T_{n-1 \mid \mathcal{N}}^{\circ}\right)$ is a doubly commuting $(n-1)$-tuple of $p$-hyponormal operators on $\mathcal{N}$ and $\left(z_{1} a_{1}, \ldots, z_{n-1} a_{n-1}\right) \in \sigma_{T}(\mathbf{S})$, Theorem 7 of [3] and Theorem D imply that there exists a non-zero vector $y^{\circ}$ in $\mathcal{N}$ such that

$$
\left(T_{j}^{\circ}-z_{j} a_{j}\right)^{*} y^{\circ}=0 \text { for every } j(j=1, \ldots, n-1) .
$$

Let

$$
\mathcal{L}=\bigcap_{j=1}^{n-1} \operatorname{Ker}\left(\left(T_{j}^{\circ}-z_{j} a_{j}\right)^{*}\right) .
$$

Then $\mathcal{N} \cap \mathcal{L}$ is a non-zero subspace of $\mathcal{K}$. Hence we have $\left(z_{n}, a_{n}\right) \in \sigma_{j p}\left(U_{n \mid \mathcal{L}}^{\circ}, A_{n \mid \mathcal{L}}^{\circ}\right)$ and $\left(z_{n}, a_{n}\right) \in \sigma_{X}\left(U_{n \mid \mathcal{L}}^{\circ},\left|T_{n \mid \mathcal{L}}\right|^{\circ}\right)$. Also by the induction we have

$$
z_{n} a_{n} \in \sigma\left(T_{n \mid \mathcal{L}}^{\circ}\right) .
$$

Since $T_{n \mid \mathcal{L}}^{\circ}$ is a $p$-hyponormal operator on $\mathcal{L}$, there exists a non-zero vector $w^{\circ} \in \mathcal{L}$ such that

$$
\left(T_{n}^{\circ}-z_{n} a_{n}\right)^{*} w^{\circ}=0 .
$$

Therefore, there exists a sequence $\left\{x_{m}\right\}$ of unit vectors such that

$$
\left(T_{j}-z_{j} a_{j}\right)^{*} x_{m} \rightarrow 0 \text { for every } j(j=1, \ldots, n) .
$$

Hence we have $\left(z_{1} a_{1}, \ldots, z_{n} a_{n}\right) \in \sigma_{T}(\mathbf{T})$.

Conversely, we assume that $\left(z_{1} a_{1}, \ldots, z_{n} a_{n}\right) \in \sigma_{T}(\mathbf{T})$. Also assume that the theorem holds for doubly commuting $(n-1)$-tuples of $p$-hyponormal operators. By Theorem 7 of $\left[3\right.$ there exists a sequence $\left\{x_{m}\right\}$ of unit vectors such that

$$
\left(T_{j}-z_{j} a_{j}\right)^{*} x_{m} \rightarrow 0 \text { for every } j(j=1, \ldots, n) .
$$

Consider the extension space $\mathcal{K}$ of $\mathcal{H}$ and let

$$
\mathcal{U}=\operatorname{Ker}\left(\left(T_{n}^{\circ}-z_{n} a_{n}\right)^{*}\right) .
$$

By Theorem $D$ and (1) there exists $z^{\circ} \in \mathcal{U}$ such that

$$
\left(T_{j}^{\circ}-z_{j} a_{j}\right)^{*} z^{\circ}=0 \text { for every } j(j=1, \ldots, n-1) .
$$


Since $\left(T_{1}^{\circ}, \ldots, T_{n-1}^{\circ}\right)$ is a commuting $(n-1)$-tuple of $p$-hyponormal operators on $\mathcal{U}$, it holds that $\left(z_{1} a_{1}, \ldots, z_{n-1} a_{n-1}\right) \in \sigma_{T}\left(T_{1 \mid \mathcal{U}}^{\circ}, \ldots, T_{n-1 \mid \mathcal{U}}^{\circ}\right)$. By the inductive hypothesis

$$
\left(z_{1}, \ldots, z_{n-1}, a_{1} \cdots a_{n-1}\right) \in \sigma_{X}\left(\mathbf{U}^{\prime}, \mathbf{A}^{\prime}\right),
$$

where $\mathbf{U}^{\prime}=\left(U_{1 \mid \mathcal{U}}^{\circ}, \ldots, U_{n-1 \mid \mathcal{U}}^{\circ}\right)$ and $A^{\prime}=\left|T_{1 \mid \mathcal{U}}^{\circ}\right| \cdots\left|T_{n-1 \mid \mathcal{U}}^{\circ}\right|$. Hence there exist $\left(m_{1}, \ldots, m_{n-1}\right) \in[0,1]^{n-1}$ and a non-zero vector $u^{\circ} \in \mathcal{U}$ such that

$$
\left(U_{j}^{\circ}-z_{j}\right) u^{\circ}=\left(A_{1}^{\circ} \cdots A_{n-1}^{\circ}-a_{1} \cdots a_{n-1}\right) u^{\circ}=0,
$$

where $A_{j}=\left\{m_{j} \mathcal{S}_{j}^{+}\left(\left|T_{j}\right|^{2 p}\right)+\left(1-m_{j}\right) \mathcal{S}_{j}^{-}\left(\left|T_{j}\right|^{2 p}\right)\right\}^{\frac{1}{2 p}}$ for every $j(j=1, \ldots, n-1)$. Next let

$$
\mathcal{V}=\bigcap_{j=1}^{n-1} \operatorname{Ker}\left(U_{j}^{\circ}-z_{j}\right) \cap \operatorname{Ker}\left(A_{1}^{\circ} \cdots A_{n-1}^{\circ}-a_{1} \cdots a_{n-1}\right) .
$$

Since $\mathcal{U} \cap \mathcal{V}$ is a non-zero subspace, we have

$$
z_{n} a_{n} \in \sigma\left(T_{n \mid \mathcal{V}}^{\circ}\right) \text {. }
$$

Hence by Theorem 1 there exists $0 \leq m_{n} \leq 1$ such that $z_{n} a_{n} \in \sigma\left(U_{n} A_{n}\right)$, where $A_{n}=\left\{m_{n} \mathcal{S}_{n}^{+}\left(\left|T_{n}\right|^{2 p}\right)+\left(1-m_{n}\right) \mathcal{S}_{n}^{-}\left(\left|T_{n}\right|^{2 p}\right)\right\}^{\frac{1}{2 p}}$. Since $U_{n} A_{n}$ is a normal operator,

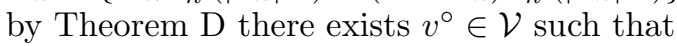

$$
\left(U_{n}^{\circ}-z_{n}\right) v^{\circ}=\left(A_{n}^{\circ}-a_{n}\right) v^{\circ}=0 .
$$

Let $\mathbf{m}=\left(m_{1}, \ldots, m_{n}\right)$ and $A_{\mathbf{m}}=\prod_{j=1}^{n} A_{j}$. By Theorem D we have

$$
\left(z_{1}, \ldots, z_{n}, a_{1} \cdots a_{n}\right) \in \sigma_{p}\left(U_{1}^{\circ}, \ldots, U_{n}^{\circ}, A_{\mathbf{m}}^{\circ}\right),
$$

and hence $\left(z_{1}, \ldots, z_{n}, a_{1} \cdots a_{n}\right) \in \sigma_{j a}\left(\mathbf{U}, A_{\mathbf{m}}\right)$. Using the definition of the Xia spectrum, we obtain

$$
\left(z_{1}, \ldots, z_{n}, a_{1} \cdots a_{n}\right) \in \sigma_{X}(\mathbf{U}, A) .
$$

The proof is now complete.

\section{ACKNOWLEDGMENTS}

This paper was inspired by the paper 9 and the author would like to express his cordial thanks to Professor B. P. Duggal. He would also like to express his thanks to Professor Raul E. Cruto for his useful suggestion.

\section{REFERENCES}

[1] S. K. Berberian, Approximate proper vectors, Proc. Amer. Math. Soc. 13(1962), 111-114. MR 24:A3516

[2] J. Bunce, The joint spectrum of commuting nonnormal operators, Proc. Amer. Math. Soc. 29(1971), 499-505. MR 44:832

[3] M. Chō, Spectral properties of p-hyponormal operators, Glasgow Math. J. 36(1994), 117-122. MR 94m:47043

[4] M. Chō and M. Itoh, Putnam's inequality for p-hyponormal operators, Proc. Amer. Math. Soc. 123(1995), 2435-2440. MR 95j:47027

[5] M. Chō and M. Itoh, On spectra of p-hyponormal operators, Integr. Equat. Oper. Th. 23(1995), 287-293. MR 96i:47041

[6] M. Chō and T. Huruya, Putnam's inequality for $p$-hyponormal $n$-tuples, Glasgow Math. J. 41(1999), 13-17. 
[7] R. Curto, On the connectedness of invertible $n$-tuples, Indiana Univ. Math. J. 29(1980), 393-406. MR 81e:47035

[8] R. Curto, P. Muhly and D. Xia, A trace estimate for $p$-hyponormal operators, Integr. Equat. Oper. Th. 6(1983), 507-514. MR 85b:47029

[9] B. P. Duggal, A Putnam area inequality for the spectrum of $n$-tuples of $p$-hyponormal operators, Glasgow Math. J. to appear.

[10] C. R. Putnam, Commutation properties of Hilbert space operators, Springer-Verlag, 1967. MR 36:707

[11] J. L. Taylor, A joint spectrum for several commuting operators, J. Funct. Anal. 6(1970), 172-191. MR 42:3603

[12] D. Xia, On the semi-hyponormal $n$-tuple of operators, Integr. Equat. Oper. Th. 6(1983), 879-898. MR 85b:47030

[13] D. Xia, Spectral Theory of Hyponormal Operators, Birkhäuser 1983. MR 87j:47036

Department of Mathematics, Kanagawa University, Yokohama 221-8686, Japan

E-mail address: m-cho@cc.kanagawa-u.ac.jp 